\title{
Pathogenesis of Arrhythmogenic Cardiomyopathy
}

\author{
Angeliki Asimaki, PhD, Andre G. Kleber, MD, and Jeffrey E. Saffitz, MD, PhD \\ Department of Pathology, Beth Israel Deaconess Medical Center and Harvard Medical School, \\ Boston, MA 02215, USA
}

\begin{abstract}
Arrhythmogenic cardiomyopathy (ACM) is a primary myocardial disease. It is characterized by frequent ventricular arrhythmias and increased risk of sudden cardiac death typically arising as an early manifestation before the onset of significant myocardial remodeling. Myocardial degeneration, often confined to the right ventricular free wall, with replacement by fibrofatty scar tissue, develops in many patients. ACM is a familial disease but genetic penetrance can be low and disease expression is highly variable. Inflammation may promote disease progression. It also appears that exercise increases disease penetrance and accelerates its development. More than $60 \%$ of probands harbor mutations in genes encoding desmosomal proteins, which has raised the possibility that defective cell-cell adhesion may play a role in disease pathogenesis. Recent advances have implicated changes in the canonical Wnt/ $\beta$-catenin and Hippo signaling pathways and defects in forwarding trafficking of ion channels and other proteins to the intercalated disk in cardiac myocytes. This review summarizes current understanding of the pathogenesis of ACM and highlights future research directions.
\end{abstract}

\section{Natural History of Arrhythmogenic Cardiomyopathy}

It was not until 1982 that Frank Marcus and Guy Fontaine reported the first detailed description of 24 patients with what they called "arrhythmogenic right ventricular dysplasia" (ARVD) which, they postulated, originated as a developmental anomaly of the right ventricle. ${ }^{1}$ It has since been recognized that this disease falls within the spectrum of the nonischemic cardiomyopathies as classified by the World Health Organization in $1994 .{ }^{2}$ The designation "arrhythmogenic right ventricular cardiomyopathy" (ARVC) reflected the preferential involvement of right ventricle in many cases. However, the more recent recognition of left dominant and biventricular forms has led to adoption of the broader term "arrhythmogenic cardiomyopathy" (ACM). ${ }^{3}$

ACM is estimated to affect 1:1000 - 1:5000 individuals in the general population. ${ }^{4}$ It is among the most common causes of sudden cardiac death (SCD) in people $\leq 35$ years of age. ${ }^{3}$ The actual prevalence may be greater because the diagnosis may be missed clinically and at

\footnotetext{
Address correspondence to: Jeffrey E. Saffitz, MD, PhD, Department of Pathology, Beth Israel Deaconess Medical Center, 330 Brookline Avenue, Boston, MA 02215, Phone: 617-667-4343, Fax: 617-667-2943, jsaffitz@ bidmc.harvard.edu.

Publisher's Disclaimer: This is a PDF file of an unedited manuscript that has been accepted for publication. As a service to our customers we are providing this early version of the manuscript. The manuscript will undergo copyediting, typesetting, and review of the resulting proof before it is published in its final citable form. Please note that during the production process errors may be discovered which could affect the content, and all legal disclaimers that apply to the journal pertain.
} 
postmortem examination. Its cardinal feature is the early predisposition to ventricular arrhythmias and SCD typically arising in the context of well-preserved ventricular structure and function. Thus, in its early stages, ACM is more reminiscent of the ion channelopathies than the other non-ischemic cardiomyopathies. ${ }^{5}$

As the disease evolves, the ventricular myocardium exhibits progressive degeneration of cardiac myocytes with replacement by fat and fibrous tissue. The pattern and distribution of myocardial degeneration are highly characteristic and distinctly different than that seen in other cardiomyopathies. ${ }^{6}$ In contrast to the tendency for greater involvement of subendocardial muscle in various forms of heart disease associated with hypertrophy, it is the subepicardium of the RV free wall that is most commonly affected in ACM. Moreover, segments of the RV free wall that experience the greatest mechanical stress during the cardiac cycle - the posterior segment below the tricuspid annulus, the apex and the RV outflow tract - show the greatest damage. In general, the endocardial trabeculated muscles of the RV and the interventricular septum are spared. When the left ventricle is involved, myocardial degeneration and fibrosis are most conspicuous in the subepicardium and midmyocardium of the lateral free wall. ${ }^{6}$

Originally, two pathological forms of the disease were proposed: fatty and the fibro-fatty. ${ }^{7}$ However, it is now recognized that significant ingrowth of epicardial adipocytes may occur in the RV in elderly or obese individuals which, in extreme cases, can extend focally to the endocardial surface and at least superficially resemble the picture seen in ACM. Therefore, infiltration of adipose tissue without inflammation and myocyte degeneration is no longer considered diagnostic of ACM. Instead, histologic features of myocyte degeneration/death and fibrosis, with or without fat, in a specific distribution, are considered the most reliable histopathological indicators of the disease. ${ }^{6}$ Another typical feature is mononuclear inflammation, mainly lymphocytic, which may be extensive and associated with areas of myocyte degeneration creating a picture similar to that seen in viral myocarditis. The role of inflammation in ACM remains largely undefined. It has been proposed that inflammatory infiltrates may extend injury to previously unaffected regions of the myocardium, a process associated with episodic exacerbations characterized by more severe ECG abnormalities increased symptomatic arrhythmias causing dizziness, palpitations, and syncope. Such a clinical picture may be misdiagnosed as myocarditis. ${ }^{3}$

The natural history of classical right-sided ACM is divided into four stages. In the initial or concealed phase, there are minimal or no structural abnormalities. Yet, the patient is at risk of SCD. The second phase is characterized by ventricular arrhythmias of left bundle branch block morphology and structural changes, typically confined to the RV, which can be observed using echocardiography, angiography and/or magnetic resonance imaging. During this phase, patients may experience syncope and/or palpitations. The third phase is defined by overt RV failure with overall preserved LV function. The final phase is marked by overt LV involvement and biventricular heart failure. Left dominant ACM is characterized by primary (but not necessarily exclusive) LV involvement, while biventricular ACM is defined by early and parallel involvement of both ventricles. ${ }^{3}$ 
The clinical diagnosis of ACM remains challenging owing to its age-related progression, vast phenotypic variation and incomplete penetrance. No single gold-standard diagnostic test exists. Instead, diagnosis relies on a scoring system first introduced in 1994 with "major" and "minor" criteria from categories including structural changes, ECG changes, family history, arrhythmias and tissue pathology. ${ }^{2}$ Although these so-called "Task Force criteria" are relatively specific, they are not highly sensitive. Diagnostic power was increased in the revised 2010 criteria in which included genetic criteria and more quantification of metrics allowing for greater discrimination and identification of earlier/milder forms of ACM. ${ }^{8}$

A number of diseases may mimic ACM rendering its diagnosis even more challenging. Early ACM can be misdiagnosed as idiopathic RVOT tachycardia ${ }^{9}$ or Brugada syndrome. ${ }^{10}$ Nevertheless, current research indicates that idiopathic RVOT tachycardia may not be as benign as originally thought. ${ }^{11}$ There is also evidence that Brugada syndrome may also involve structural abnormalities of the RVOT. ${ }^{12}$ In the advanced biventricular form, ACM is almost impossible to distinguish from dilated cardiomyopathy (DCM). As mentioned above, ACM may also mimic myocarditis and vice-versa. ${ }^{13}$ Sarcoidosis is a well-known phenocopy of ACM. Patients with sarcoid may present with many features considered to be highly diagnostic of ACM including inverted T-waves in right precordial leads, ventricular tachycardia of LBBB morphology, RV abnormalities seen by various imaging modalities, late potentials, and $>2000$ premature ventricular contractions on $24 \mathrm{hr}-H o l t e r$ monitoring. Differential diagnosis typically relies on a heart biopsy showing non-caseating granulomas or other features of sarcoidosis not seen in ACM including hilar lymphadenopathy with uptake of ${ }^{22} \mathrm{~F}$-2deoxy-glucose on positron emission tomography. ${ }^{14}$ Other pathologies that mimic ACM include pulmonary hypertension and Uhl's anomaly. The revised Task Force Criteria provide cut-off values for RV imaging and histopathological changes, which facilitates differential diagnosis. ${ }^{8}$

That exercise may lead to more adverse events in ACM has been recognized for many years but until recently the evidence has been largely circumstantial. ${ }^{15}$ In 1995 Marcus and Fontaine reported that SCD in subjects with ARVC often occurs during exercise. ${ }^{16}$ They attributed this to the fact that exercise causes significant stretch of the RV and a disproportionate increase in RV afterload following exercise, which, they thought promoted ventricular arrhythmias. ${ }^{17}$ A subsequent study published in 2005 from Johns Hopkins University reported that physical activity was part of the daily life of $49 \%$ of their ACM patients, $50 \%$ of whom were female. ${ }^{18}$ The striking male predominance in the disease in Europe could be related at least in part to greater proportion of men engaged in exercise in Europe. ${ }^{19}$ A larger study conducted at Johns Hopkins showed that earlier disease onset (under 25 years of age) was significantly associated with intense exercise. The same study also suggested that exercise could be a trigger for disease manifestation in previously unaffected mutation carriers and could accelerate disease progression. ${ }^{20}$

ACM management is focused mainly on SCD prevention. Young competitive athletes with ACM have a 5.4-fold increased risk of SCD compared to non-athletes. ${ }^{21}$ Both probands and relatives manifesting disease-related abnormalities are, therefore, advised to avoid competitive sports and endurance training. Implantable cardiac defibrillators (ICD) are being used increasingly for primary prevention in cases of definite ACM. Antiarrhythmic 
pharmacological agents as well as catheter ablation are also used to reduce the arrhythmic burden. Cardiac transplantation, though relatively unusual in this disease spectrum, is an option in patients with incessant VT or end-stage HF. ${ }^{3}$

\section{Genetics of Arrhythmogenic Cardiomyopathy}

Adherens junctions and desmosomes are intercellular adhesion complexes which, in cardiac myocytes, reside within the intercalated disk. Classically, desmosomes are thought to contain members of the cadherin family (desmoglein2 and desmocollin2), which link adjacent cells, and members of the plakin and armadillo families (plakoglobin, plakophilin2 and desmoplakin), which connect the desmosomal plaque to the intermediate filaments of the cytoskeleton. ${ }^{22}$ In cardiac adherens junctions the major cadherin molecule linking adjacent cells is $\mathrm{N}$-cadherin while the intracellular plaque proteins include plakoglobin, $\beta$ catenin and a-catenin, which connect the junction to the actin cytoskeleton. Recent studies, however, have shown that the two junction structures are not in fact distinct in terms of their molecular composition. Rather, "classical adherens junction proteins" are found in apparent desmosomes and vice versa. Moreover, a-catenin has been shown to interact with plakophilin-2 thus creating a hybrid, enforced junction structure termed the area composita (Fig 1). ${ }^{3}$

Identification of the genetic basis of ACM was hampered by difficulties in accurately identifying affected and non-affected individuals within families related to low penetrance and variable disease expression. It is not surprising, therefore, that initial breakthroughs came from studies of rare recessive syndromes in which penetrance was very high and disease expression unequivocal. The first such discovery of a causative mutation came from studies of patients with Naxos disease, a recessive syndrome consisting of the triad of typical right-sided ACM (ARVC), palmoplantar keratoderma and wooly hair. Linkage analysis mapped the disease locus to $17 \mathrm{q} 21^{24}$ and the causative mutation was identified as a two base-pair deletion (2057del2) in the gene encoding the desmosomal protein plakoglobin $(J U P) .^{25}$

Today we know that $>60 \%$ of ACM patients bear mutations in one or more of the five major desmosomal genes (encoding plakoglobin, desmoplakin, plakophilin2, desmoglein2 and desmocollin2). In the great majority of cases, inheritance is autosomal dominant. The ARVC database, created by the University Medical Center Groningen in the Netherlands (www.arvcdatabase.info), currently lists $>1,400$ unique desmosomal gene variants identified in ACM patients worldwide. ${ }^{26}$ It is noteworthy that severe or earlier onset cases of the disease are often caused by compound (two alleles for the same gene bear different mutations) or digenic heterozygosity (mutations in two different genes, for instance a mutation in the gene encoding plakophilin2 and a mutation in the gene encoding desmoglein 2$).{ }^{26}$

ACM has also been associated with mutations in non-desmosomal genes. A variant in the cardiac ryanodine receptor gene ( $R Y R 2)$ was originally linked to a distinct form of ACM (ARVC2) characterized by juvenile SCD and effort-induced polymorphic VT. ${ }^{27}$ Further studies, however, suggested that ARVC2 may, in fact, be a form of catecholaminergic 
polymorphic VT, and does not belong to the spectrum of ACM. ${ }^{28}$ The S358L mutation in the gene encoding transmembrane protein 43 (TMEM43), identified in a founder population in Newfoundland, Canada, causes a fully penetrant, non-classical form of ACM characterized by a high incidence of premature SCD and severe HF in survivors. ${ }^{29}$ TMEM43 is localized at the inner nuclear membrane. It contains a large hydrophilic domain that extends into the endoplasmic reticulum and is believed to maintain the integrity of the inner nuclear membrane through interactions with other proteins such as lamins and emerin. Mutations in the laminA/B gene have been implicated in DCM and mutations in emerin have been associated with Emery-Dreifuss muscular dystrophy. ${ }^{30}$ Since TMEM43 affects the distribution of emerin, it is thought that perhaps mutations in the TMEM43 gene could cause muscular dystrophy. ${ }^{31}$ TMEM43 also contains a response element for peroxisome proliferator-activated receptor gamma (PPAR $\gamma$ ), an adipogenic transcription factor, which could possibly be related to adipogenesis in ACM. ${ }^{28}$ Another non-desmosomal gene implicated in ACM is phospholamban $(P L N)$, which regulates the sarcoplasmic reticulum calcium pump (SERCA2) and hence plays a key role in $\mathrm{Ca}^{+2}$ homeostasis. The R14del mutation in $P L N$ was first identified in patients with DCM but more recently has been identified in patients in a Dutch cohort with a clinical and pathological phenotype of ACM. ${ }^{31}$ Another ACM locus mapped to chromosome 14q24.3 apparently involves the gene encoding transforming growth factor 3 beta $(T G F 3 \beta)$ which is known to play a role in cardiac inflammation and fibrosis. Although no mutations have been identified within the coding region of the $T G F 3 \beta$ gene in patients with $\mathrm{ACM}$, variants within the promoter region (c. $36 \mathrm{G}>\mathrm{A}$ and c. $1723 \mathrm{C}>\mathrm{T}$ ) have been documented in 2 families. ${ }^{32}$ In vitro studies suggest that these changes increase the activity of the TGF3 $\beta$ gene by at least two-fold. ${ }^{32}$

Additionally, two mutations in the gene encoding the cardiac intermediate filament protein desmin have been identified in patients with $\mathrm{ACM} .{ }^{33}$ One patient bearing the K241E mutation in DES had a severe form of ACM but was also a carrier of a mutation in the desmosomal protein plakophilin2. The second patient carried the A213V mutation in DES, which has previously been associated with DCM. ${ }^{33}$ Finally, a mutation $(1051 \mathrm{~A}>\mathrm{G})$ in the $L D B 3$ gene (encoding the desmin/dystrophin complex protein Cypher /ZASP) was recently identified in an ACM family of Spanish origin. ${ }^{34}$ Mutations in this gene have previously been linked to various clinical phenotypes including DCM, HCM or certain forms of myofibrillar myopathy. ${ }^{34}$

\section{Variable clinical and pathological expression of ACM disease alleles}

Despite progress in identifying disease alleles in ACM, molecular mechanisms underlying the highly variable genotype-phenotype relationships in this disease are poorly understood. Typically, the disease and an underlying mutation are identified in a family following the sudden death of a proband. Subsequent genetic testing often identifies first degree relatives who carry the same variant implicated in the proband's demise but who exhibit no clinical evidence of disease. Similarly, little is known about how a single variant can produce different clinical disease phenotypes. ${ }^{35}$ As mentioned above, for example, the R14del mutation in $P L N^{31}$ and the A213V mutation in $D E S^{32}$ can cause either ACM or DCM phenotypes. Mutations in Cypher/ZASP can cause DCM, HCM and ACM. ${ }^{34}$ Mutations in desmoplakin can cause either typical right-sided ARVC or left dominant forms of the 
disease. ${ }^{36}$ Understanding the genetic basis and the role of environmental modifiers responsible for these interesting observations is a subject of great interest for future research.

\section{Role of abnormal cell-cell adhesion in ACM}

Even before desmosomal gene mutations had been linked to the pathogenesis of ACM, an electron microscopy study had reported changes in cardiac intercalated disks which were described as pale and flattened and in which desmosomes were rare, small and/or absent in several disks. While provocative, this study lacked rigorous morphometric analysis. ${ }^{37}$ Several years later, Basso et al. performed an ultrastructural investigation of myocardial biopsy samples obtained from 21 ACM patients. They reported reduced numbers of desmosomes which were thought to be elongated and misplaced compared to that seen in DCM or control samples. While this study did not include quantitative analysis, it did raise an important question about whether a defect in cell-cell adhesion, reflected by ultrastructural remodeling of desmosomes, plays a role in ACM. ${ }^{38}$ Electron microscopic analysis has also been performed on hearts of transgenic mice with cardiac-specific expression of desmoglein2 bearing the N271S mutation. These studies, in which tissue preservation artifacts can be minimized, reported widening of specific regions of the intercalated disk and loss of desmosomes in areas of myocardial injury. ${ }^{39}$

However, even if intercalated disks are remodeled in ACM and they develop "gaps" or regions of apparent cell-cell separation, the question remains whether disease pathogenesis is related to abnormalities in cell-cell adhesion. The answer requires actual measurements of the strength of cell-cell adhesion in cardiac myocytes expressing disease alleles. In 2011, Sato et al. investigated the effects of siRNA-mediated silencing of the gene encoding the desmosomal protein plakophilin2 (PKP2) on cell-cell adhesion in monolayers of neonatal rat ventricular myocytes in vitro. ${ }^{40}$ They used an assay in which a confluent monolayer of adherent cells is incubated with dispase, an enzyme that cleaves proteins connecting the cells to the underlying substrate without interfering with proteins responsible for cell-cell connections. As a result, the intact monolayer lifts from the culture dish and can be subjected to a defined mechanical intervention. Sato et al. reported that following a brief interval of agitation, monolayers of cells in which PKP2 was silenced broke apart into a greater number of fragments than control monolayers, indicating reduced cell-cell adhesion. ${ }^{40}$ In a recent study from our laboratory, we confirmed the findings of Sato et al. and also showed that silencing expression of plakoglobin, another desmosomal protein, in neonatal rat ventricular myocytes also leads to reduced cell-cell adhesion. ${ }^{41}$ By contrast, however, when monolayers of neonatal rat ventricular myocytes expressed mutant forms of either $P K P 2$ or JUP, no such reduction in intercellular adhesion could be detected. Rather, cells expressing mutant desmosomal proteins exhibited abnormal signaling in response to mechanical intervention. ${ }^{41}$ These results raise questions about a primary role for reduced cell-cell adhesion in ACM. They also highlight biological differences produced by silencing expression of a desmosomal protein vs. expression of a mutant form of the protein. Finally, they focus attention on potential abnormalities in how the heart responds to mechanical stress in ACM which has clear implications regarding exercise as a determinant of disease expression and severity. 


\section{Molecular Pathology of ACM}

To gain further insights into disease mechanisms, we have characterized the distribution of intercalated disk proteins in myocardial samples from $>100$ patients with dominantly inherited ACM. In nearly every case, the amount of immunoreactive signal for the desmosomal protein plakoglobin is depressed at cell-cell junctions (Fig 2). ${ }^{42}$ As discussed below, cellular redistribution of plakoglobin appears to be a hallmark of ACM and is likely to play a key role in its pathogenesis. This finding has been confirmed in independent studies and has been seen in association with mutations in all of the desmosomal genes as well as in patients who fulfill diagnostic criteria for ACM but in whom no desmosomal mutation can be detected. ${ }^{43,44}$ Reduced plakoglobin signal at intercalated disks has also been reported in ACM patients from Newfoundland bearing the S358L mutation in TMEM $43^{45}$ and ACM patients from the Netherlands bearing the R14del mutation in PLN (but not in patients with the same $P L N$ mutation but who present with a DCM phenotype). ${ }^{31}$ Thus, reduced junctional signal for plakoglobin appears to track with the disease phenotype.

Reduced plakoglobin signal at intercalated disks is generally not seen in patients with ischemic heart disease or other forms of non-ischemic cardiomyopathy. ${ }^{42}$ However, reduced plakoglobin signal has been documented in cases of sarcoidosis and giant cell myocarditis, both granulomatous forms of myocarditis associated with malignant ventricular arrhythmias. ${ }^{46}$ In vitro studies have shown that incubation of neonatal rat ventricular myocytes with inflammatory cytokines highly expressed in sarcoidosis and giant cell myocarditis (including IL-17, IL-6 and TNFa) causes a rapid shift of plakoglobin from junctional to cytoplasmic/nuclear pools. ${ }^{46}$ Furthermore, ACM patients have increased circulating levels of selected inflammatory mediators including IL-6R, IL-8, monocyte chemoattractant protein 1 and macrophage inflammatory protein $1 \beta .{ }^{46}$ Collectively, these observations suggest potential mechanistic links between ACM and highly arrhythmogenic forms of granulomatous myocarditis and offer new insights perhaps explaining clinical similarities between ACM and cardiac sarcoidosis. They also raise the possibility that inflammatory mediators may play a direct role in the pathogenesis of ACM.

\section{The potential role of plakoglobin in intracellular signaling}

Plakoglobin ( $\gamma$-catenin) is a member of the armadillo protein family and contains 13 central repeats (so-called Armadillo repeats) of 42 amino acids flanked by distinct $\mathrm{N}$ - and Cterminal regions. Although closely related, the armadillo protein $\beta$-catenin cannot replace plakoglobin at desmosomal sites. ${ }^{47,48}$ Only plakoglobin links desmosomal cadherins to intermediate filaments of the cytoskeleton via interactions with desmoplakin, whereas both plakoglobin and $\beta$-catenin link $\mathrm{N}$ - or E-cadherins to the actin cytoskeleton through $\alpha$-catenin (Fig 1). ${ }^{47,48}$ The mechanism responsible for redistribution of plakoglobin from the junctional pool to intracellular and nuclear pools in ACM has not been fully elucidated. We and others hypothesize that in ACM plakoglobin fails to integrate into the desmosome/fascia adherens complex because desmosomal assembly is disturbed, ${ }^{47,} 48$ but exactly how that happens is not understood. 
In normal cells, $\beta$-catenin fulfills a dual function as a structural protein in composite junctions and as a regulated signaling molecule. ${ }^{49-52}$ By contrast, plakoglobin or $\gamma$-catenin is almost entirely sequestered and confined to the cell-cell junctions and its potential role as a signaling molecule is much less well known. ${ }^{53-55}$ The balance between junctional and nuclear pools of $\beta$-catenin is regulated by GSK-3 $\beta$. In turn, GSK-3 $\beta$ activity is strongly modulated by Wnt signaling. Phosphorylation of $\beta$-catenin by this kinase, which is present in a GSK-3 $\beta /$ axin/APC/CK complex, makes it accessible to ubiquitin ligase ( $\beta$-TrCP) thus promoting its degradation. ${ }^{53-55}$ If plakoglobin is overexpressed, or its distribution is altered as apparently occurs in ACM, it may serve as an additional substrate of GSK-3 $\beta$ / axin/APC/CK. ${ }^{49,53-55}$ Moreover, the degradation of plakoglobin along the proteasomal pathway is slower than degradation of $\beta$-catenin. ${ }^{51,55-57}$ These observations raise the possibility that redistribution of plakoglobin from intercalated disks to the myocyte nucleus in ACM may interfere with transcriptional activity regulated by the $\beta$-catenin/Tcf/Lef complex. ${ }^{51}$ In fact, suppression of nuclear $\beta$-catenin/Wnt signaling and other features of the ACM phenotype have been observed following overexpression of wild type plakoglobin in normal cardiac myocytes. ${ }^{51,56}$ However, the extent to which interactions between $\beta$-catenin and plakoglobin within the nucleus are responsible for the pathogenesis in ACM is not known. It is also unknown whether abnormal trafficking of $\mathrm{Cx} 43$ and ion channels in $\mathrm{ACM}^{44}$ is related to disturbance of nuclear $\beta$-catenin-dependent transcription or occurs by an independent mechanism. This question is considered below.

\section{Gap junction and ion channel remodeling in ACM}

Formation and maintenance of functional gap junctions depends on the presence of stable mechanical connections between neighboring cells. ${ }^{57}$ Thus, it is perhaps not surprising that gap junction remodeling, shown by reduced immunoreactive signal for the major ventricular gap junction protein Cx43, occurs in the great majority of cases of ACM (Fig 3A). ${ }^{42}$

Nevertheless, neither the responsible mechanism nor the pathophysiological consequence of gap junction remodeling in ACM is known. It does appear, however, that reduced junctional expression of Cx43 occurs early in ACM, during the concealed phase, when arrhythmias develop in hearts that show little if any structural remodeling. By contrast, gap junction remodeling in other forms of heart disease often occurs as a late feature in myocardium exhibiting variable degrees of hypertrophy, myocytolytic degeneration and fibrosis. This distinction suggests that a different mechanism may apply to gap junction remodeling in ACM. ${ }^{5}$ In any event, the potential role of electrical uncoupling in slowing of electrical propagation, arrhythmogenesis and SCD in ACM remains to be determined. This question takes on additional importance in view of recent findings indicating that the cardiac sodium current, $\mathrm{I}_{\mathrm{Na}} 40,58,59$ and the inward rectifier current, $\mathrm{I}_{\mathrm{K} 1}, 58$ are reduced in experimental models of ACM. It is well known that impulse propagation in myocardium is critically dependent on the extent of cell-cell electrical coupling at gap junctions, the amount of depolarizing current generated by $\mathrm{I}_{\mathrm{Na}}$ and the level of the resting membrane potential dependent on the inward rectifier current $\mathrm{I}_{\mathrm{K} 1} \cdot{ }^{60}$ The combination of reduced electrical coupling and reduced $\mathrm{I}_{\mathrm{Na}}$ and $\mathrm{I}_{\mathrm{K} 1}$ could, therefore, have a significant impact on electrical propagation and arrhythmogenesis. Thus far, however, evidence of reduced $\mathrm{I}_{\mathrm{Na}}$ in patients with ACM is indirect and consists of reduced immunoreactive signal at intercalated disks for 
Nav1.5, the major protein subunit of the sodium current (Fig 3B) ${ }^{61}$ Potential mechanisms leading to decreased $\mathrm{I}_{\mathrm{Na}}$ and $\mathrm{I}_{\mathrm{K} 1}$ flux are discussed below. Future work will be required to characterize arrhythmia mechanisms related to changes in cellular electrophysiology in the concealed phase and to define the contribution of tissue remodeling on arrhythmias during more advanced stages of the disease in which macroscopic conduction pathways are altered by myocardial degeneration and fibrosis.

\section{Aberrant protein trafficking in ACM}

Despite marked changes in the distribution of plakoglobin, $\mathrm{Cx} 43$ and Nav1.5 in various models of ACM, there is no apparent difference in the total cellular amount of these proteins. ${ }^{58}$ These observations point to a general defect in protein trafficking rather than a change in protein synthesis and/or degradation. ${ }^{58}$ However, the exact mechanisms responsible for disturbed channel trafficking in ACM are unknown. Trafficking of $\mathrm{Cx} 43$ connexons occurs along microtubular highways which interact with actin, and connect dynamically to the peripheral targets via the plus-end protein EB1. ${ }^{62-64}$ Cargo delivery occurs at adherens junctions, where the presence of p150 (Glued) and $\beta$-catenin are required for $\mathrm{Cx} 43$ delivery to the intercalated disc. ${ }^{62}$ Moreover, EB1 interacts with the desmosomal protein desmoplakin. ${ }^{65}$ Analysis of experimental models of ACM by super-resolution microscopy has shown that the average distance between EB1 and N-cadherin is increased. ${ }^{59}$ There is also evidence that the distribution of microtubules and the tipping of microtubules by EB1 are abnormal in experimental models, ${ }^{66}$ suggesting a disturbance in the entire EB1-dependent trafficking apparatus in ACM. Relatively little is known about trafficking of Nav1.5 and Kir2.1 to specific pools within the intercalated disc. Both Nav1.5 $\left(\mathrm{I}_{\mathrm{Na}}\right)$ and $\mathrm{Kir} 2.1\left(\mathrm{I}_{\mathrm{K} 1}\right)$, as well as Kv4.3 ( $\mathrm{I}_{\mathrm{to}}$ ) have C-terminal Ser-Ile-Val, (SIV) motifs which bind to the PDZ domains of the MAGUK protein SAP97. As deduced from experiments involving silencing its expression, SAP97 appears to regulate Nav1.567 and the stoichiometry of Nav1.5 and Kir2.1 ${ }^{68}$ at the intercalated disc, and it has been suggested that the channels together with SAP97 traffic as a multi-protein complex. ${ }^{68}$ Recently however, it has been shown that localization of Nav1.5 at the intercalated disc is unaffected in a mouse model with genetic ablation of the PDZ-binding domain on the C-terminal of Nav1.5 (SIV motif). ${ }^{69}$ Moreover, cardiac myocyte-specific deletion of SAP97 in mice is associated with reduced $\mathrm{I}_{\mathrm{K} 1}, \mathrm{I}_{\mathrm{to}}$ and $\mathrm{I}_{\mathrm{Kur}}$, but not $\mathrm{I}_{\mathrm{Na}}{ }^{70}$ At present, discrepancies between effects of silencing SAP97 expression versus specific genetic ablation of channel SIV motifs have not been resolved and the exact functional role of SAP97 remains undefined.

We have reported that immunoreactive signal for SAP97 is depressed at intercalated disks in myocardium from patients with ACM but not in other forms of heart disease. ${ }^{58}$ Junctional signal for SAP97 is also reduced in neonatal rat ventricular myocytes transfected to express mutant forms of plakoglobin or plakophilin2 known to cause ACM in patients. ${ }^{58}$ To further investigate the role of SAP97 in ACM, we used shRNA to silence its expression in neonatal rat ventricular myocytes and examined the distribution of intercalated proteins by immunohistochemistry. We confirmed the previously reported reduction in Nav1.5 at cellcell junctions. ${ }^{67} \mathrm{We}$ also observed that localization of plakoglobin was greatly reduced whereas the distribution of other mechanical junction proteins such as $\mathrm{N}$-cadherin and plakophilin2 and the gap junction protein $\mathrm{Cx} 43$ was unaffected (Fig 4). ${ }^{58}$ Thus, while 
SAP97 reduction at the intercalated disc seems to be a consistent feature of ACM, further investigations are needed to clarify its role in protein trafficking defects that appear to be important in ACM pathogenesis.

\section{Possible mechanisms for lipo- and fibrogenesis in ACM}

The first evidence in support of the "dysregulated signaling" hypothesis in ACM came from Marian and colleagues who showed that knockdown of desmoplakin expression in HL-1 cells leads to nuclear translocation of plakoglobin, suppression of the canonical Wnt signaling pathway and increased expression of fibrogenic and adipogenic genes. ${ }^{47}$

Desmoplakin-deficient cells were also shown to contain lipid droplets consistent with activation of adipogenic pathways. Although a direct causal relationship between nuclear accumulation of plakoglobin and altered Wnt signaling was not established, this study provided the first evidence of dysregulated Wnt signaling in the pathogenesis of ACM. The same group then investigated the source of excess adipocytes in ACM. ${ }^{71}$ Using genetic fatemapping methods, they showed that a subset of second heart field cardiac progenitor cells is driven by plakoglobin to follow an adipogenic fate. When plakoglobin expression is knocked down, these progenitor cells resist differentiation into adipocytes whereas in the presence of plakoglobin, they express increased mRNA levels of the Wnt-regulated genes Krüppel-like factor 15 (KLF15) and insulin-like growth factor-binding protein 5 (IGFBP5). $K L F 15$ is highly expressed in adipose tissue and is up-regulated during the differentiation of pre-adipocytes into adipocytes. IGFBP5 plays an important role in a variety of pathophysiological settings including muscle atrophy, cell death, fibrosis and inflammation. ${ }^{71}$ Marian's studies, therefore, provided significant new evidence of dysregulated signaling, perhaps mediated by plakoglobin, which could promote inflammation and fibroadiposis in ACM.

The Hippo signaling pathway includes the scaffold protein Salvador (SAV) and the mammalian STE20-like kinase 1 (MST1), MST2, large tumor suppressor 1 (LATS1) and LATS2. Activation of this pathway results in the phosphorylation, inactivation and nuclear exclusion of the Hippo-effector molecule Yes-associated protein (YAP). In the nucleus, YAP interacts with $\beta$-catenin to drive Wnt-related gene expression. Recently, Chen et al. reported that in myocardial samples from patients with ACM as well as in experimental models, plakoglobin redistribution leads to aberrant activation of the Hippo pathway, translocation of YAP to junctional sites and further suppression of the canonical Wnt signaling pathway contributing to myocyte death and fibroadipogenesis. ${ }^{72}$ This important study was the first demonstration of altered Wnt signaling in patient myocardial samples.

\section{Mechanistic insights from drug screening}

To gain further insights into disease mechanisms, we collaborated with Calum MacRae at Brigham and Women's Hospital to produce a transgenic zebrafish model of ACM with cardiac-specific expression of plakoglobin with the 2057del2 mutation. Larval fish showed bradycardia, reduced cardiac output and reduced stroke volume by 48 hours post fertilization. Young adult fish developed ventricular dilatation, cachexia and early mortality. ${ }^{58}$ Using this model, MacRae and colleagues screened a library of bioactive 
compounds and found that one compound in particular, SB216763, had a remarkable ability to prevent bradycardia and contractility defects and normalize survival. Subsequent studies showed that SB216763 also reversed action potential abnormalities and normalized $\mathrm{I}_{\mathrm{Na}}$ and $\mathrm{I}_{\mathrm{K} 1}$ current densities in ventricular myocytes isolated from the mutant fish. ${ }^{58} \mathrm{SB} 216763$ has previously been annotated as an inhibitor of GSK3 $\beta$ and hence an activator of the canonical Wnt signaling pathway. ${ }^{73}$ These observations are, therefore, consistent with previous evidence from Marian implicating dysregulation of Wnt signaling in the pathogenesis of ACM.

We have performed additional studies in neonatal rat ventricular myocytes expressing mutant forms of plakoglobin or plakophilin2 implicated in human disease. As seen in ACM patient samples, key intercalated disk proteins including plakoglobin, Cx43, Nav1.5 and SAP97 fail to localize normally in the transfected cells. ${ }^{58}$ These in vitro models also show increased myocyte apoptosis (which is exacerbated by cyclical stretch or shear stress), increased expression of inflammatory mediators, and pathologic changes in action potential morphology (reduced action potential upstroke velocity and reduced resting membrane potential) consistent with reduced $\mathrm{I}_{\mathrm{Na}}$ and $\mathrm{I}_{\mathrm{K} 1}$ current densities. All of these changes are reversed by incubating cells with SB216763 for 24 hours (Fig 5). ${ }^{58}$ The rapidity with which these defects are reversed by SB216763 provides further support for the notion that defective protein trafficking rather than abnormal protein production plays an important role in ACM. These observations also suggest that both the arrhythmia and cell injury phenotypes in ACM are mediated by a common disease mechanism which is responsive to a single small molecule inhibitor independent of the specific disease allele that causes the disease. ${ }^{58}$

In further support of this hypothesis, Marian has reported that that treatment of cardiac progenitor cells with 6-bromoindirubin-3'-oxime (BIO), another GSK3 $\beta$ inhibitor/Wnt activator, inhibits up-regulation of KLF15 and IGFBP5 and prevents cells from differentiating into adipocytes. ${ }^{71}$ Additionally, Kim et al. showed that BIO prevents lipogenesis, redistribution of plakoglobin redistribution, apoptosis and calcium handling defects in iPSC-derived cardiac myocytes obtained from ACM patients with PKP2 mutations. $^{74}$

\section{Conclusions}

The pathogenesis of ACM is complex. The low genetic penetrance, age-related disease progression and variable clinical expression all speak to powerful effects of as yet poorly defined genetic, epigenetic and environmental modifiers (one of which appears to be exercise). The fact that ACM is caused by mutations in genes encoding desmosomal proteins has appropriately focused attention on intercellular junctions and cell-cell adhesion, but recent advances have converged on a developing paradigm of abnormal Wnt and Hippo signaling, stimulated by mechanical stress, which interferes with forward trafficking of key proteins to the cell surface, promotes fibrogenic and adipogenic gene expression profiles, and perhaps induces an immune response involving multiple inflammatory mediators. The fact that a single small molecule, SB216763, can reverse both the cell injury and arrhythmia phenotypes in multiple ACM models provides convincing evidence for a common disease 
mechanism. Whether this drug targets GSK3 $\beta$ and/or other molecules remains to be proven. Nevertheless, we now have a more complete picture and a deeper underlying of molecular mechanisms in ACM than for the other non-ischemic cardiomyopathies. Much work remains to be done to improve earlier detection, risk stratification and prevention of sudden death.

\section{Glossary}

$\mathrm{ACM}$

ARVD

ARVC

HCM

DCM

SCD

RVOT

RV

LBBB

ICD

JUP

RYR2

TMEM43

PLN

SERCA2

TGF3 $\beta$

DES

LDB3

PKP2

IL-17

IL-6

TNFa

IL-6R

IL-8

Cx43

GSK3 $\beta$

APC arrhythmogenic cardiomyopathy

arrhythmogenic right ventricular dysplasia

arrhythmogenic right ventricular cardiomyopathy

hypertrophic cardiomyopathy

dilated cardiomyopathy

sudden cardiac death

right ventricular outflow tract

right ventricle

left bundle branch block

implantable cardiac defibrillator

gene encoding plakoglobin

gene encoding the cardiac ryanodine receptor

transmembrane protein 43

Phospholamban

gene encoding the sarcoplasmic reticulum calcium pump

transforming growth factor 3 beta

gene encoding desmin

gene encoding the desmin/dystrophin complex protein Cypher/ZASP

plakophilin2

interleukin-17

interleukin-6

tumor necrosis factor alpha

interleukin-6 receptor

interleukin-8

connexin43

glycogen synthase kinase 3 beta

adenomatous polyposis coli 


$\begin{array}{ll}\text { KLF15 } & \text { Krüppel-like factor 15 } \\ \text { IGFBP5 } & \text { insulin-like growth factor binding protein 5 } \\ \text { MST1/2 } & \text { mammalian STE20-like kinase 1/2 } \\ \text { LATS1/2 } & \text { large tumor suppressor 1/2 } \\ \text { YAP } & \text { Yes-associated protein } \\ \text { MAGUK } & \text { membrane-associated guanylate kinase } \\ \text { SAP97 } & \text { synapse-associated protein 97 } \\ \text { BIO } & \text { 6-bromoindirubin-3'-oxime } \\ \text { SIV } & \text { residues Ser-Ile-Val, SIV }\end{array}$

\section{References}

1. Marcus FI, Fontaine GH, Guiraudon G, et al. Right ventricular dysplasia: a report of 24 adult cases. Circulation. 1982; 65:384-398. [PubMed: 7053899]

2. McKenna WJ, Thiene G, Nava A, et al. Diagnosis of arrhythmogenic right ventricular dysplasia/ cardiomyopathy. Task Force of the Working Group Myocardial and Pericardial Disease of the European Society of Cardiology and of the Scientific Council on Cardiomyopathies of the International Society and Federation of Cardiology. Br Heart J. 1994; 71:215-218. [PubMed: 8142187]

3. Sen-Chowdhry S, Morgan RD, Chambers JC, McKenna WJ. Arrhythmogenic cardiomyopathy: etiology, diagnosis and treatment. Ann Rev Med. 2010; 61:233-253. [PubMed: 20059337]

4. Quartaa, g; Elliott, PM. Diagnostic Criteria for Arrhythmogenic Right Ventricular Cardiomyopathy. Rev Esp Cardiol. 2012; 65:599-605. [PubMed: 22622265]

5. Saffitz JE, Asimaki A, Huang H. Arrhythmogenic right ventricular cardiomyopathy: new insights into mechanisms of disease. Cardiovasc Pathol. 2010; 19:166-170. [PubMed: 20051321]

6. Basso C, Thiene G. Adipositas cordis, fatty infiltration of the right ventricle, and arrhythmogenic right ventricular cardiomyopathy. Just a matter of fat? Cardiovasc Pathol. 2005; 14:37-41. [PubMed: 15710290]

7. Basso C, Thiene G, Corrado D, et al. Arrhythmogenic right ventricular cardiomyopathy. Dysplasia, dystrophy, or myocarditis? Circulation. 1996; 94:983-991. [PubMed: 8790036]

8. Marcus FI, McKenna WJ, Sherrill D, et al. Diagnosis of arrhythmogenic right ventricular cardiomyopathy/ dysplasia: proposed modification of the task force criteria. Circulation. 2010; 121:1533-1541. [PubMed: 20172911]

9. Ren L, et al. Electrocardiographic difference between ventricular arrhythmias from the right ventricular outflow tract and idiopathic right ventricular arrhythmias. Pacing Clin Electrophysiol. 2014; 37:1658-1664. [PubMed: 25081355]

10. Agullo-Pascual E, Cerrone M, Delmar M. Arrhythmogenic cardiomyopathy and Brugada syndrome: diseases of the connexome. FEBS Lett. 2014; 588:1322-1330. [PubMed: 24548564]

11. Viskin S, Rosso R, Rogowski O, Belhassen B. The "short-coupled" variant of right ventricular outflow ventricular tachycardia: a not-so-benign form of benign ventricular tachycardia? J Cardiovasc Electrophysiol. 2005; 16:912-916. [PubMed: 16101636]

12. Catalano O, et al. Magnetic resonance investigations in Brugada syndrome reveal unexpectedly high rate of structural abnormalities. Eur Heart J. 2009; 30:2241-2248. [PubMed: 19561025]

13. Ellinor PT, MacRae CA, Thierfelder L. Arrhythmogenic right ventricular cardiomyopathy. Heart Fail Clin. 2010; 6:161-177. [PubMed: 20347785]

14. Ott P, Marcus FI, Sobonya RE, et al. Cardiac sarcoidosis masquerading as right ventricular dysplasia. Pacing Clin Electrophysiol. 2003; 26:1498-1503. [PubMed: 12914628] 
15. Rojas A, Calkins H. Present understanding of the relationship between exercise and arrhythmogenic right ventricular dysplasia/cardiomyopathy. Trends Cardiovasc Med. 2014 pii: S1050-1738 [Epub ahead of print].

16. Marcus FI, Fontaine G. Arrhythmogenic right ventricular dysplasia/cardiomyopathy: a review. Pacing Clin Electrophysiol. 1995; 18:1298-1314. [PubMed: 7659585]

17. Marcus FI, Zareba W, Calkins H, et al. Arrhythmogenic right ventricular cardiomyopathy/ dysplasia, clinical presentation and diagnostic evaluation: results from the North American multidisciplinary study. Heart Rhythm. 2009; 6:984-992. [PubMed: 19560088]

18. Dalal D, Nasir K, Bomma C, et al. Arrhythmogenic right ventricular dysplasia: a United States experience. Circulation. 2005; 112:3823-3832. [PubMed: 16344387]

19. den Haan AD, Tan BY, Zikusoka MN, et al. Comprehensive desmosome mutation analysis in North Americans with Arrhythmogenic right ventricular dysplasia/cardiomyopathy. Circ Cardiovasc Genet. 2009; 2:428-435. [PubMed: 20031617]

20. James CA, Bhonsale A, Tichnell C, et al. Exercise increases age-related penetrance and arrhythmic risk in arrhythmogenic right ventricular dysplasia/cardiomyopathy-associated desmosomal mutation carriers. J Am Coll Cardiol. 2013; 62:1290-1297. [PubMed: 23871885]

21. Sen-Chowdhry S, McKenna WJ. Sudden death from genetic and acquired cardiomyopathies. Circulation. 2012; 125:1563-1576. [PubMed: 22451606]

22. Green KJ, Gaudry CA. Are desmosomes more than tethers for intermediate filaments. Nat Rev Mol Cell Biol. 2000; 1:208-216. [PubMed: 11252896]

23. Li J, Radice GL. A new perspective on intercalated disk organization: implications for heart disease. Dermatol Res Pract. 2010 Article ID 207835.

24. Coonar AS, Protonotarios N, Tsatsopoulou A, et al. Gene for arrhythmogenic right ventricular cardiomyopathy with diffuse nonepidermolytic palmoplantar keratoderma and woolly hair (Naxos disease) maps to 17q21. Circulation. 1998; 97:2049-2058. [PubMed: 9610536]

25. McKoy G, Protonotarios N, Crosby A, et al. Identification of a deletion in plakoglobin in arrhythmogenic right ventricular cardiomyopathy with palmoplantar keratoderma and woolly hair (Naxos disease). Lancet. 2000; 355:2119-2124. [PubMed: 10902626]

26. Lazzarini E, Jongbloed JD, Pilichou K, et al. The ARVD/C Genetic Variants Database: 2014 Update. Hum Mutat. 2015 [Epub ahead of print].

27. Tiso N, Stephan DA, Nava A, et al. Identification of mutations in the cardiac ryanodine receptor gene in families affected with arrhythmogenic right ventricular cardiomyopathy type 2 (ARVD2). Hum Mol Genet. 2001; 10:189-194. [PubMed: 11159936]

28. George CH, Higgs GV, Lai FA. Ryanodine receptor mutations associated with stress-induced ventricular tachycardia mediate increased calcium release in stimulated cardiomyocytes. Circ Res. 2003; 93:531-540. [PubMed: 12919952]

29. Merner ND, Hodgkinson KA, Haywood AF, et al. Arrhythmogenic right ventricular cardiomyopathy type 5 is a fully penetrant, lethal arrhythmic disorder caused by a missense mutation in the TMEM43 gene. Am J Hum Genet. 2008; 82:809-821. [PubMed: 18313022]

30. Rajkumar R, Sembrat JC, McDonough B, et al. Functional effects of the TMEM43 Ser358Leu mutation in the pathogenesis of arrhythmogenic right ventricular cardiomyopathy. BMC Med Genet. 2012; 13:21. [PubMed: 22458570]

31. van der Zwaag PA1, van Rijsingen IA, Asimaki A, et al. Phospholamban R14del mutation in patients diagnosed with dilated cardiomyopathy or arrhythmogenic right ventricular cardiomyopathy: evidence supporting the concept of arrhythmogenic cardiomyopathy. Eur J Heart Fail. 2012; 14:1199-1207. [PubMed: 22820313]

32. Beffagna G, Occhi G, Nava A, et al. Regulatory mutations in transforming growth factor-beta3 gene cause arrhythmogenic right ventricular cardiomyopathy type 1. Cardiovasc Res. 2005; 65:366-373. [PubMed: 15639475]

33. Lorenzon A, Beffagna G, Bauce B, et al. Desmin mutations and arrhythmogenic right ventricular cardiomyopathy. Am J Cardiol. 2013; 111:400-405. [PubMed: 23168288]

34. Lopez-Ayala JM, Ortiz-Genga M, Gomez-Milanes I, et al. A mutation in the Z-line Cypher/ZASP protein is associated with arrhythmogenic right ventricular cardiomyopathy. Clin Genet. 2014 [Epub ahead of print]. 
35. Marcus FI, Edson S, Towbin JA. Genetics of arrhythmogenic right ventricular cardiomyopathy: a practical guide for physicians. J Am Coll Cardiol. 2013; 61:1945-1948. [PubMed: 23500315]

36. Rizzo S, Pilichou K, Thiene G, Basso C. The changing spectrum of arrhythmogenic (right ventricular) cardiomyopathy. Cell Tissue Res. 2012; 348:319-323. [PubMed: 22476658]

37. Guiraudon CM. Histological diagnosis of right ventricular dysplasia: a role for electron microscopy? Eur Heart J. 1989; 10(Suppl D):95-96. [PubMed: 2806313]

38. Basso C, Czarnowska E, Della Barbera M, et al. Ultrastructural evidence of intercalated disc remodelling in arrhythmogenic right ventricular cardiomyopathy: an electron microscopy investigation on endomyocardial biopsies. Eur Heart J. 2006; 27:1847-1854. [PubMed: 16774985]

39. Pilichou K, Remme CA, Basso C, et al. Myocyte necrosis underlies progressive myocardial dystrophy in mouse dsg2-related arrhythmogenic right ventricular cardiomyopathy. J Exp Med. 2009; 206:1787-1802. [PubMed: 19635863]

40. Sato PY, Coombs W, Lin X, et al. Interactions between ankyrin-G, Plakophilin-2, and Connexin43 at the cardiac intercalated disc. Circ Res. 2011; 109:193-201. [PubMed: 21617128]

41. Hariharan V, Asimaki A, Michaelson JE, et al. Arrhythmogenic right ventricular cardiomyopathy mutations alter shear response without changes in cell-cell adhesion. Cardiovasc Res. 2014; 104:280-289. [PubMed: 25253076]

42. Asimaki A, Tandri H, Huang H, et al. A new diagnostic test for arrhythmogenic right ventricular cardiomyopathy. N Engl J Med. 2009; 360:1075-1084. [PubMed: 19279339]

43. Munkholm J, Andersen CB, Ottesen GL. Plakoglobin: A diagnostic marker of arrhythmogenic right ventricular cardiomyopathy in forensic pathology? Forensic Sci Med Pathol. 2015; 11:47-52. [PubMed: 25549959]

44. Ermakov S, Ursell PC, Johnson CJ, et al. Plakoglobin immunolocalization as a diagnostic test for arrhythmogenic right ventricular cardiomyopathy. Pacing Clin Electrophysiol. 2014; 37:17081716. [PubMed: 25196244]

45. Siragam V, Cui X, Masse S, et al. TMEM43 mutation p.S358L alters intercalated disc protein expression and reduces conduction velocity in arrhythmogenic right ventricular cardiomyopathy. PLoS One. 2014; 9:e109128. [PubMed: 25343256]

46. Asimaki A, Tandri H, Duffy ER, et al. Altered desmosomal proteins in granulomatous myocarditis and potential pathogenic links to arrhythmogenic right ventricular cardiomyopathy. Circ Arrhythm Electrophysiol. 2011; 4:743-752. [PubMed: 21859801]

47. Garcia-Gras E, Lombardi R, Giocondo MJ, et al. Suppression of canonical Wnt/beta-catenin signaling by nuclear plakoglobin recapitulates phenotype of arrhythmogenic right ventricular cardiomyopathy. J Clin Invest. 2006; 116:2012-2021. [PubMed: 16823493]

48. Swope D, Li J, Radice GL. Beyond cell adhesion: the role of armadillo proteins in the heart. Cell Signal. 2013; 25:93-100. [PubMed: 23022961]

49. Salomon D, Sacco PA, Roy SG, et al. Regulation of beta-catenin levels and localization by overexpression of plakoglobin and inhibition of the ubiquitin-proteasome system. The Journal of cell biology. 1997; 139:1325-1335. [PubMed: 9382877]

50. Ben-Ze'ev A, Geiger B. Differential molecular interactions of beta-catenin and plakoglobin in adhesion, signaling and cancer. Current opinion in cell biology. 1998; 10:629-639. [PubMed: 9818174]

51. Choi HJ, Gross JC, Pokutta S, et al. Interactions of plakoglobin and beta-catenin with desmosomal cadherins: basis of selective exclusion of alpha- and beta-catenin from desmosomes. J Biol Chem. 2009; 284:31776-31788. [PubMed: 19759396]

52. Wahl, JKr; Nieset, JE.; Sacco-Bubulya, PA., et al. The amino- and carboxyl-terminal tails of (beta)-catenin reduce its affinity for desmoglein 2. Journal of cell science. 2000; 113:1737-1745. Pt 10. [PubMed: 10769205]

53. Sadot E, Simcha I, Iwai K, et al. Differential interaction of plakoglobin and beta-catenin with the ubiquitin-proteasome system. Oncogene. 2000; 19:1992-2001. [PubMed: 10803460]

54. Zhurinsky J, Shtutman M, Ben-Ze'ev A. Plakoglobin and beta-catenin: protein interactions, regulation and biological roles. Journal of cell science. 2000; 113:3127-3139. Pt 18. [PubMed: 10954412] 
55. Williams BO, Barish GD, Klymkowsky MW, et al. A comparative evaluation of beta-catenin and plakoglobin signaling activity. Oncogene. 2000; 19:5720-5728. [PubMed: 11126358]

56. Lombardi R, da Graca Cabreira-Hansen M, Bell A, et al. Nuclear plakoglobin is essential for differentiation of cardiac progenitor cells to adipocytes in arrhythmogenic right ventricular cardiomyopathy. Circ Res. 2011; 109:1342-1353. [PubMed: 22021931]

57. Saffitz JE. Dependence of electrical coupling on mechanical coupling in cardiac myocytes insights gained from cardiomyopathies caused by defects in cell-cell communication. Ann NY Acad Sci. 2005; 1047:336-344. [PubMed: 16093509]

58. Asimaki A, Kapoor S, Plovie E, et al. Identification of a new modulator of the intercalated disc in a zebrafish model of arrhythmogenic cardiomyopathy. Sci Transl Med. 2014; 6:240ra74.

59. Cerrone M, Noorman M, Lin X, et al. Sodium current deficit and arrhythmogenesis in a murine model of plakophilin-2 haploinsufficiency. Cardiovasc Res. 2012; 95:460-468. [PubMed: 22764151]

60. Kléber AG, Rudy Y. Basic mechanisms of cardiac impulse propagation and associated arrhythmias. Physiol Rev. 2004; 84:431-488. [PubMed: 15044680]

61. Noorman M, Hakim S, Kessler E, et al. Remodeling of the cardiac sodium channel, connexin43, and plakoglobin at the intercalated disk in patients with arrhythmogenic cardiomyopathy. Heart Rhythm. 2013; 10:412-419. [PubMed: 23178689]

62. Shaw RM, Fay AJ, Puthenveedu MA, et al. Microtubule plus-end-tracking proteins target gap junctions directly from the cell interior to adherens junctions. Cell. 2007; 128:547-560. [PubMed: 17289573]

63. Smyth JW, et al. Actin cytoskeleton rest stops regulate anterograde traffic of connexin 43 vesicles to the plasma membrane. Circ Res. 2012; 110:978-989. [PubMed: 22328533]

64. Smyth JW, Shaw RM. Visualizing cardiac ion channel trafficking pathways. Methods Enzymol. 2012; 505:187-202. [PubMed: 22289454]

65. Patel DM, Dubash AD, Kreitzer G, Green KJ. Disease mutations in desmoplakin inhibit Cx43 membrane targeting mediated by desmoplakin-EB1 interactions. J Cell Biol. 2014; 206:779-797. [PubMed: 25225338]

66. Zhang S, Kuhn DA, Kessler E, Asimaki A, Sottas V, Vanhecke D, Rothen-Rutishauser B, Saffitz JE, Shaw RM, Kleber AG. Arrhythmogenic Cardiomyopathy Mutations Cause Disassembly of the Cx43 Forward Trafficking Machinery Which Can Be Rescued by GSK-3 $\beta$ Inhibition. Circulation. 2014; 130(suppl.):A15042.

67. Petitprez S, Zmoos AF, Ogrodnik J, et al. SAP97 and dystrophin macromolecular complexes determine two pools of cardiac sodium channels Nav1.5 in cardiomyocytes. Circ Res. 2011; 108:294-304. [PubMed: 21164104]

68. Milstein ML1, Musa H, Balbuena DP, et al. Dynamic reciprocity of sodium and potassium channel expression in a macromolecular complex controls cardiac excitability and arrhythmia. Proc Natl Acad Sci U S A. 2012; 109:E2134-2143. [PubMed: 22509027]

69. Shy D, Gillet L, Ogrodnik J, Albesa M, Verkerk AO, Wolswinkel R, Rougier JS, Barc J, Essers MC, Syam N, Marsman RF, Van Mil AM, Rotman S, Redon R, Bezzina CR, Remme CA, Abriel H. PDZ domain-binding motif regulates cardiomyocyte compartment-specific Nav1.5 channel expression and function. Circulation. 2014; 130:147-160. [PubMed: 24895455]

70. Gillet L, Rougier JS, Shy D, Sonntag S, Mougenot N, Essers M, Shmerling D, Balse E, Hatem SN, Abriel H. Cardiac-specific ablation of synapse-associated protein SAP97 in mice decreases potassium currents but not sodium current. Heart Rhythm. 2015; 12:181-192. [PubMed: 25447080]

71. Lombardi R, Dong J, Rodriguez G, et al. Genetic fate mapping identifies second heart field progenitor cells as a source of adipocytes in arrhythmogenic right ventricular cardiomyopathy. Circ Res. 2009; 104:1076-1084. [PubMed: 19359597]

72. Chen SN, Gurha P, Lombardi R, et al. The hippo pathway is activated and is a causal mechanism for adipogenesis in arrhythmogenic cardiomyopathy. Circ Res. 2014; 114:454-468. [PubMed: 24276085] 
73. Zhu Z, Kremer P, Tadmori I, et al. Lithium suppresses astrogliogenesis by neural stem and progenitor cells by inhibiting STAT3 pathway independently of glycogen synthase kinase 3 beta. PLoS One. 2011; 6:e23341. [PubMed: 21931595]

74. Kim C, Wong J, Wen J, et al. Studying arrhythmogenic right ventricular dysplasia with patientspecific iPSCs. Nature. 2013; 494:105-110. [PubMed: 23354045]

75. Asimaki A, Kléber AG, MacRae CA, Saffitz JE. Arrhythmogenic Cardiomyopathy - New Insights into Disease Mechanisms and Drug Discovery. Prog Pediatr Cardiol. 2014; 37:3-7. [PubMed: 25506190] 


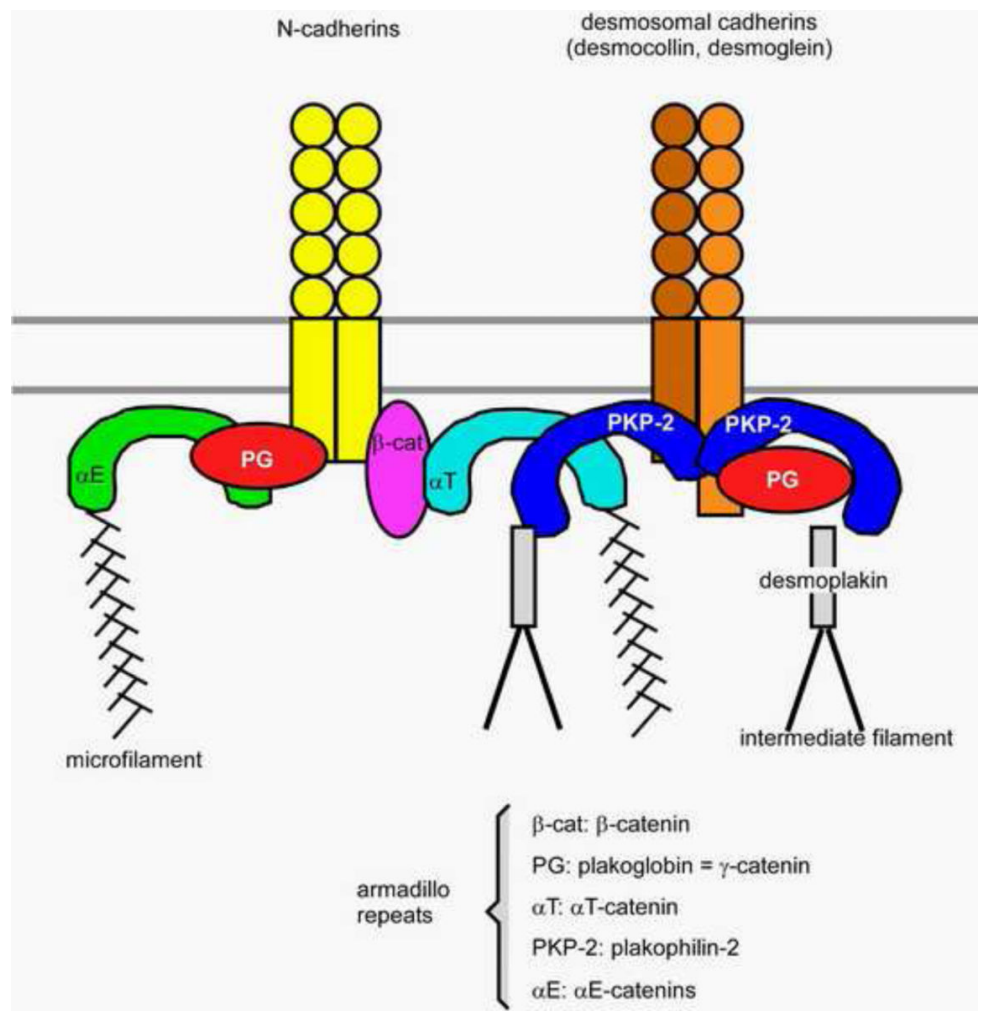

Figure 1.

Schematic diagram of area composita: adherens junction and desmosomal proteins form a hybrid enforced junction structure that is attached to both the actin and the intermediate filament cytoskeleton (modified from Li and Radice. Dermatol Res Pract 2010). ${ }^{23}$ 

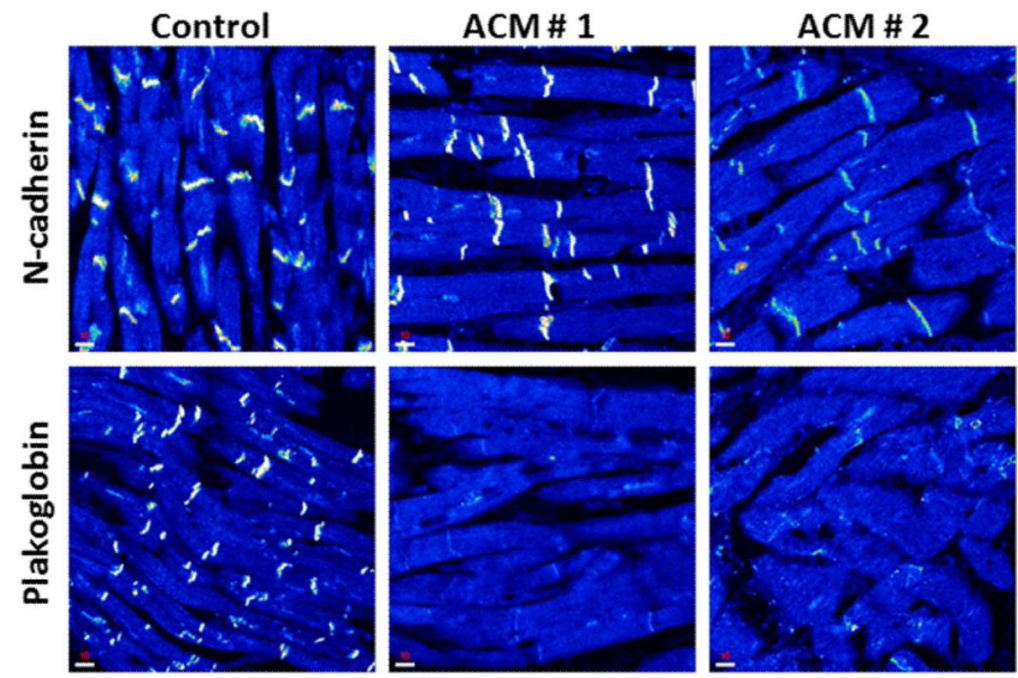

Figure 2.

Confocal immunofluorescence microscopy analysis of control and ACM left ventricular myocardium. Immunoreactive signal for plakoglobin at cardiac intercalated disks was markedly reduced in both ACM samples, while signal for the non-desmosomal protein Ncadherin was strong and indistinguishable from controls (modified from Asimaki et al. NEJM 2009). ${ }^{42}$ 


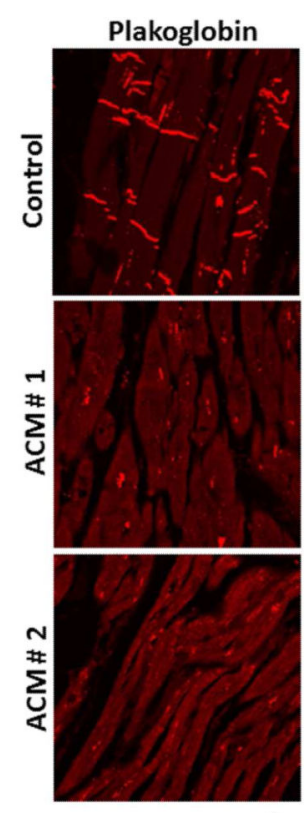

A.

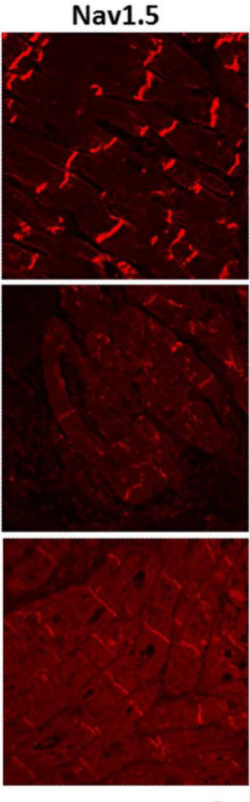

B.

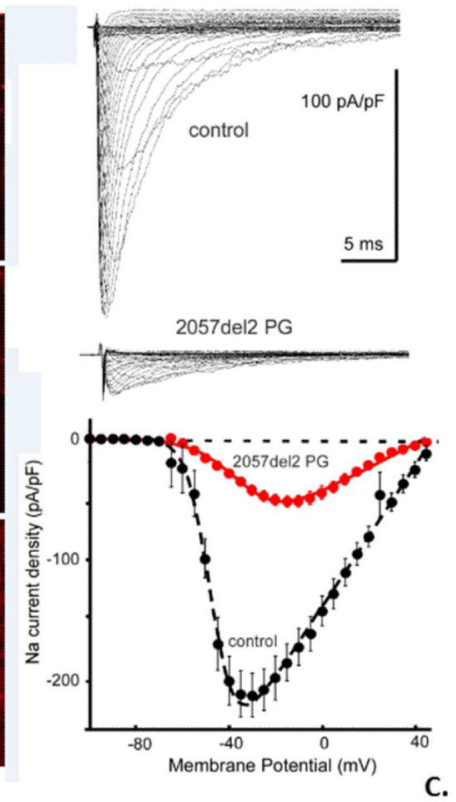

Figure 3.

Representative confocal immunofluorescence images of control ventricular myocardium and myocardium from two patients with ACM. Specific immunoreactive signal for Cx43 (A) and Nav1.5 (B) is significantly depressed at intercalated disks in both ACM cases. ${ }^{75}$ Changes in $\mathrm{I}_{\mathrm{Na}}$ current density in zebrafish myocytes expressing 2057del2 plakoglobin (C). ${ }^{58}$ 

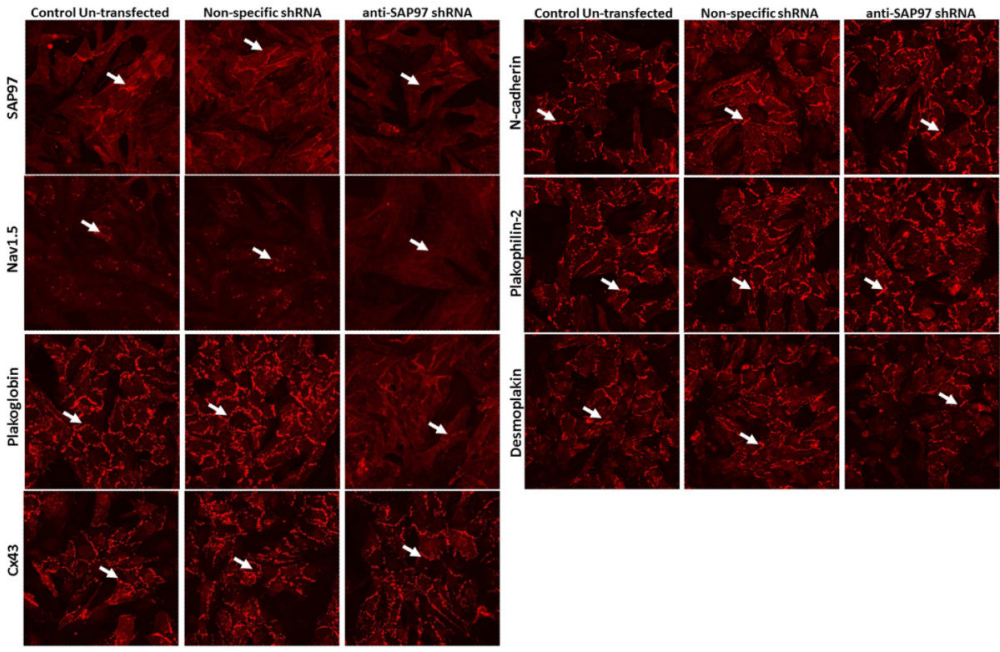

Figure 4.

Cultures of neonatal rat ventricular myocytes (NRVMs) were treated with anti-SAP97 shRNA for 72hrs. Non-specific shRNA was used as a negative control. Knock-down of SAP97 had no effect on the distribution of N-cadherin, desmoplakin, plakophilin2 and Cx43. It did, however, significantly reduce junctional signal for Nav1.5 and plakoglobin. Arrows point to immunoreactive signal concentrated at cell-cell junctions. ${ }^{58}$ 

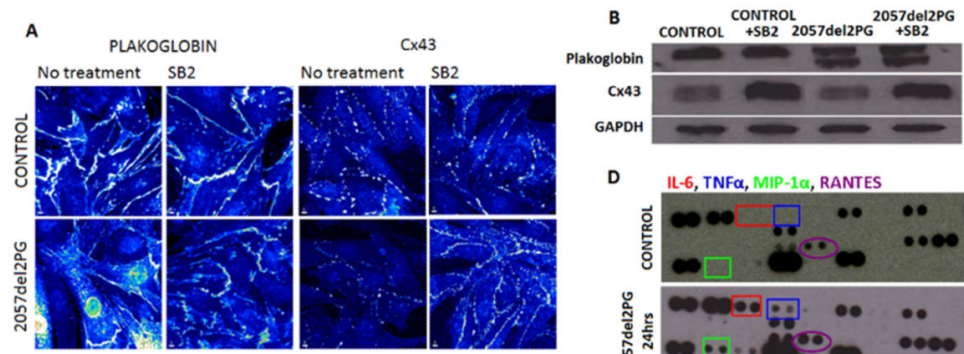

C No treatment SB2
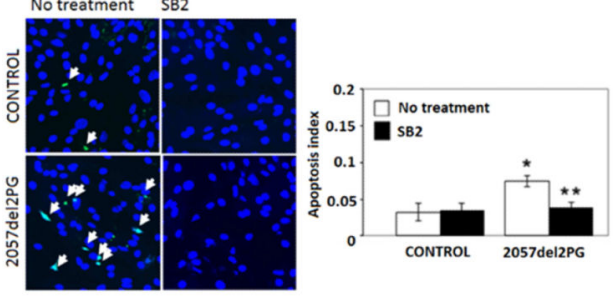

D IL-6, TNF $\alpha$, MIP-1 $\alpha$, RANTES
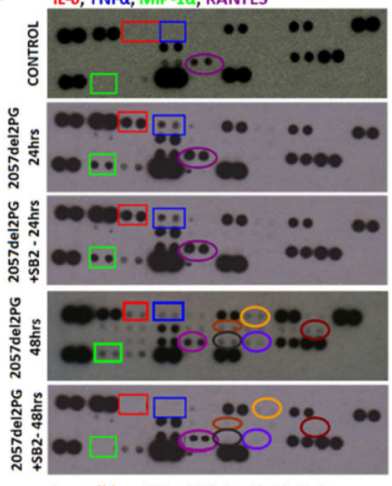

Fractalkine, IFNy, MIP-1Y, IP-10, IL-1ra

Figure 5.

Representative confocal immunofluorescence images showing restoration of localization of plakoglobin and Cx43 in NRVMs expressing a mutant form of plakoglobin (2057del2) following 24hrs treatment with SB216763 (A). Western immunoblots showing the total cellular content for plakoglobin and Cx43 in transfected NRVMs in the presence or absence of SB216763 (B). TUNEL labeling of control NRVMs and NRVMs expressing 2057del2 plakoglobin in the presence or absence of SB2. White arrow point to apoptotic nuclei. $* \mathrm{P}<0.01$ versus controls, $* * \mathrm{P}<0.001$ versus untreated transfected myocytes; 2 -tailed student's t-test (C). Effects of SB216763 on inflammatory cytokines secreted by NRVMs expressing $2057 \mathrm{del} 2$ plakoglobin (D). ${ }^{58}$ 